\title{
Chronophysiologie circadienne du cuir chevelu
}

\section{Circadian chronophysiology of the scalp}

\author{
C. Piérard-Franchimont ${ }^{\mathrm{a}, \mathrm{b}}$, F. Henry ${ }^{\mathrm{a}, \mathrm{b}}$, G. Loussouarn ${ }^{\mathrm{c}}$, D. Saint-Léger ${ }^{\mathrm{c}}$, G.-E. Piérard ${ }^{\mathrm{a}, *}$ \\ ${ }^{a}$ Service de dermatopathologie, CHU du Sart Tilman, 4000 Liège, Belgique \\ ${ }^{\mathrm{b}}$ Service de dermatologie, centre hospitalier Hutois, 4500 Huy, Belgique \\ ${ }^{\mathrm{c}}$ L'Oréal recherche, 92117 Clichy, France
}

Reçu le 28 octobre 2005 ; accepté le 9 mars 2007

Disponible sur internet le 23 mai 2007

\begin{abstract}
Résumé
Les rythmes biologiques sont nombreux et ils sont également variés selon leurs périodicités et amplitudes. Des variations au cours du jour, et en particulier des rythmes circadiens ont été rapportés au niveau de la peau du visage et des avant-bras. Cependant, peu d'information existe au niveau des biorythmes quotidiens du cuir chevelu. Le but de l'étude était d'explorer la chronophysiologie du cuir chevelu. Nous rapportons des rythmes diurnes chez huit hommes caucasiens. Ils sont révélés par la méthode des plexogrammes par intervalles de quatre heures sur une période de cinq jours. Des biorythmes statistiquement significatifs furent retrouvés pour la couleur de la peau, son hydratation, sa fonction barrière, son $\mathrm{pH}$ et l'excrétion de sébum. Le début de l'après-midi était le moment d'accroissement maximum de la rougeur, de l'hydratation, du pH et du débit sébacé. Il correspondait aussi à la diminution maximale de la fonction barrière cutanée. L'amplitude de certains de ces biorythmes était si faible que leur pertinence clinique reste incertaine, même si le caractère statistique est significatif. Compte tenu de leur synchronisme apparent, il est probable qu'un oscillateur central exerce une influence prépondérante. Cependant, rares sont les individus qui expriment des biorythmes pour tous les paramètres physiologiques considérés. La réactivité périphérique joue donc également un rôle important pour définir les bons et les mauvais répondeurs au biorythme potentiel de chaque paramètre individuel.
\end{abstract}

(C) 2007 Elsevier Masson SAS. Tous droits réservés.

\begin{abstract}
Biological rhythms are numerous and vary according to periodicity and amplitude. Variations over the course of a day, particularly circadian rhythms have been reported in the skin of the face and forearms. However, little information is available about daily biorhythms of the scalp. The aim of the present study was to explore the chronophysiology of the scalp. We report a series of diurnal rhythms in 8 Caucasian men using the plexogram method at 4-h intervals over a 5-day period. Statistically significant biorhythms were found for the colour, hydration, barrier function and $\mathrm{pH}$ of the skin, and for the sebum excretion as well. The early afternoon was the time of maximum increase in redness, hydration, $\mathrm{pH}$ and sebum output, combined with decreased skin barrier function. The amplitude of some of these biorhythms was so discrete that their clinical relevance remained uncertain even when they appeared statistically significant. In view of the apparent synchronization between them, it is likely that a central oscillator exerts a major influence. However, only rare subjects exhibited biorhythms for all the considered physiological parameters. Hence, peripheral reactivity and/or environmental synchronizers also play an important role to define the good and bad respondors to the potential biorhythms of each individual parameter.
\end{abstract}

(C) 2007 Elsevier Masson SAS. Tous droits réservés.

Mots clés : Rythme circadien ; Couleur cutanée ; pH cutané ; Hydratation cutanée ; Barrière cutanée ; Sébum

Keywords: Circadian rhythm; Skin colour; Skin pH; Skin hydration; Skin barrier; Sebum

\footnotetext{
${ }^{*}$ Auteur correspondant.

Adresses e-mail : gerald.pierard@ulg.ac.be (C. Piérard-Franchimont), gerald.pierard@ulg.ac.be (G.-E. Piérard).
} 


\section{Introduction}

On appelle biorythme tout phénomène périodique régulier et spontané survenant chez des organismes vivants. Certaines de ces activités rythmiques sont des propriétés fondamentales de la matière vivante $[1,2]$. En particulier, il existe une adaptation génétique de notre structure temporelle aux variations de l'environnement liées à la rotation axiale de la Terre en 24 heures [3]. Dès lors, la finalité de l'organisation temporelle circadienne semble être l'adaptation de l'individu aux variations périodiques et prévisibles selon une période moyenne de 24 heures [2-5]. Il existe aussi une autre périodicité importante de type circannuel à laquelle correspondent des manifestations saisonnières [6-9]. Bien d'autres biorythmes, tels que les rythmes cardiaque, respiratoire et ovarien, sont indépendants de la position de la Terre par rapport au Soleil [2,10,11].

Les biorythmes qui ont une origine endogène persistent lorsque l'organisme est placé dans un environnement stabilisé et en isolement temporel. Ils sont gouvernés par un système d'horloges biologiques internes, appelées oscillateurs ou pacemakers endogènes, qui sont essentiellement localisés dans les noyaux suprachiasmatiques $[1,2,12]$. L'organisme utilise en plus des signaux périodiques, appelés synchroniseurs, pour calibrer et remettre en phase ses oscillateurs. Dès lors, les synchroniseurs, qu'ils soient exogènes ou endogènes, modulent la périodicité, mais ne créent pas les rythmes biologiques.

Certains biorythmes importants pour l'organisme humain se déroulent sur une période de 24 heures. Ils correspondent aux rythmes circadiens et nycthéméraux [12]. Un rythme nycthéméral est nettement activé et contrôlé par la lumière, qui agit alors en tant que synchronisateur majeur. Un rythme circadien (circa : environ; die : jour) est de même période, mais il est moins sensible ou n'obéit pas à l'influence de la lumière. Des rythmes nycthéméraux superposables à l'alternance entre la luminosité diurne et l'obscurité nocturne, ont été rapportés au niveau de certains mécanismes biologiques et aspects fonctionnels de la peau.

Chez l'homme, le cycle de division des kératinocytes fluctue au cours de la journée $[13,14]$. L'amplitude maximale (acrophase $\varnothing$ ) du débit des mitoses se situe vers $1 \mathrm{~h}$, alors qu'elles sont les plus rares vers $13 \mathrm{~h}$. Les cellules précancéreuses des kératoses actiniques et celles des carcinomes cutanés ont un rythme mitotique qui peut être plus ou moins perturbé $[14,15]$. Un rythme circadien, de faible amplitude, a été aussi détecté au niveau du volume de la couche cornée, atteignant son acrophase en soirée. Il pourrait être dû à des variations du volume des cornéocytes en fonction de leur charge hydrique [16].

La température cutanée, l'intensité de la sudation, le flux de sébum et le $\mathrm{pH}$ de la couche cornée sont eux aussi soumis à des fluctuations au cours du nycthémère [17-26]. Dans tous les cas, ces rythmes ne sont pas directement influencés par la lumière. La circulation capillaire cutanée est aussi soumise à un biorythme qui atteint son acrophase au milieu de la nuit, alors que son nadir (niveau le plus faible) se situe en début d'après-midi [12]. L'amplitude du rythme du flux capillaire cutané est bien supérieure à celle des rythmes de la fréquence cardiaque et de la pression artérielle. L'érythème correspondant au volume sanguin superficiel subit aussi un rythme circadien. Il est cependant différent de celui du flux sanguin puisque ses valeurs maximales ont été rapportées à midi et à $20 \mathrm{~h}$ sur le visage [26].

$\mathrm{Au}$ cours de l'alternance entre les états de veille et de sommeil, le premier est important à connaître puisque c'est à ce moment que des traitements pourraient être apportés en tenant compte d'une éventuelle chronopharmacologie [2]. Le but de cette étude était d'explorer, en période d'éveil des individus, la partie diurne de biorythmes du cuir chevelu qui à ce jour ont été très peu étudiés. Les acrophases de veille qui ont été déterminées ne correspondent donc peut-être pas à l'acrophase $\varnothing$ des cycles nycthéméraux complets. Ces derniers surviennent en effet parfois entre la fin de la soirée et l'aube.

\section{Matériel et méthodes}

L'étude a été menée selon les principes de la déclaration d'Helsinki. Elle s'est déroulée en milieu hospitalier en période hivernale. Un consentement éclairé a été signé par les volontaires. Des mesures biométrologiques non invasives ont été effectuées sur le cuir chevelu rasé de huit hommes âgés de 26 à 35 ans (Tableau 1). Elles ont été effectuées à 4 h d'intervalles, entre 6 et $23 \mathrm{~h}$, pendant cinq jours consécutifs. Aucune évaluation n'a été réalisée en phase nocturne afin de ne pas réveiller les volontaires, ce qui aurait invalidé cette étude. Toutes les évaluations ont été réalisées sur le même site du vertex rasé cinq jours avant le début des observations. L'érythème a été quantifié par le paramètre $a^{*}$ mesuré par colorimétrie en réflectance à l'aide d'un Chroma Meter ${ }^{\circledR}$ CR 200 (Minolta, Osaka, Japon). La capacitance a été mesurée en unités arbitraires par un Corneometer ${ }^{\circledR} \mathrm{CM} 820(\mathrm{C}+\mathrm{K}$ electronic, Cologne, Allemagne). La perte insensible en eau (PIE) a été estimée à l'aide d'un Tewameter ${ }^{\circledR}(\mathrm{C}+\mathrm{K}$ electronic $)$. Le $\mathrm{pH}$ a été mesuré par le pHmeter ${ }^{\circledR}(\mathrm{C}+\mathrm{K}$ electronic $)$. Le débit instantané de sébum a été mesuré par la méthode du Sebufix ${ }^{\circledR}(C+K$ electronic). La collecte de sébum pendant 45 secondes a été couplée à l'analyse quantitative de la surface relative des gouttelettes lipidiques $(\%)$ réalisée par le Visioscan $^{\circledR}(\mathrm{C}+\mathrm{K}$ electronic). Toutes les évaluations biométrologiques ont été réalisées selon les recommandations du groupe EEMCO [27-31], et notamment dans une ambiance contrôlée en température et humidité.

Tableau 1

Propriétés physiques de la peau

\begin{tabular}{|c|c|c|}
\hline Paramètre physiologique & Appareil & Paramètre physique \\
\hline Couleur (érythème) & Chroma Meter $^{(\mathbb{R}}$ & $\mathrm{a}^{*}$ \\
\hline Hydratation & Corneometer $^{\circledR}$ & $\begin{array}{l}\text { Capacitance (unités } \\
\text { arbitraires) }\end{array}$ \\
\hline Fonction barrière & Tewameter $^{\circledR}$ & $\begin{array}{l}\text { Perte insensible d'eau } \\
\text { (g/m } \mathrm{m}^{2} \text { par heure) }\end{array}$ \\
\hline Acidité & pHmeter $^{\circledR}$ & $\mathrm{pH}$ \\
\hline Excrétion de sébum & Sebufix ${ }^{\circledR}$ & Débit instantané (\%) \\
\hline
\end{tabular}


Les résultats ont été exprimés en fonction des heures locales d'hiver en Belgique. Ils ont été analysés selon les individus, ainsi que les jours et les heures de mesures. Après vérification de l'absence de discordance des rythmes selon les jours, les résultats ont été groupés pour chaque individu, et enfin pour le groupe de volontaires tout entier, sur une période de $24 \mathrm{~h}$ pour être présentés en plexogrammes de type circadien. Pour chaque paramètre et à chaque temps d'évaluation, la moyenne (M) et l'écart type (ET) ont été déterminés. Le coefficient de variation $\left(\mathrm{V}=10^{2} \mathrm{ET}^{*} \mathrm{M}^{-1}\right)$ a été calculé. L'acrophase et le nadir des valeurs des divers paramètres ont été estimés pour la période de veille diurne des individus. La différence entre chaque acrophase de veille et le mésor (moyenne) quotidien pour le paramètre considéré a été également calculée. De plus, les variations percentuelles par rapport au mésor ont été calculées pour chaque temps d'évaluation. Une analyse Anova pour chaque paramètre a été réalisée pour chaque individu et pour le groupe de volontaires en entier. Une valeur $p$ inférieure à 0,05 a été considérée comme étant statistiquement significative.

\section{Résultats}

Notre étude montre que le cuir chevelu est le siège de certains biorythmes circadiens. Les huit sujets ont présenté au moins une manifestation circadienne statistiquement significative (Tableau 2). Seul un d'entre eux a montré des biorythmes pour les cinq paramètres physiologiques envisagés.

Un biorythme circadien de la valeur $\mathrm{a}^{*}$ correspondant à la rougeur du cuir chevelu a été mis en évidence chez $4 / 8$ sujets (Tableau 2). Pour l'ensemble du groupe, l'effet de l'heure était significatif $(p<0,001)$, indépendamment du jour d'étude. Pour la période du nycthémère considérée, l'acrophase pour $\mathrm{a}^{*}$ $(8,75 \pm 0,56)$ se situait entre 14 et $15 \mathrm{~h}$, et le nadir $(8,35 \pm 0,46)$, atteint entre 6 et $7 \mathrm{~h}$, était identique à celui $(8,35 \pm 0,51)$ présent entre 22 et $23 \mathrm{~h}$ (Fig. 1). L'acrophase était 2,8\% supérieure à la moyenne quotidienne. La moyenne des $\mathrm{V}$ intra-individuels et interjournaliers s'échelonnait entre 1,89 et $3,10 \%$ selon les heures, le maximum étant atteint entre 10 et $11 \mathrm{~h}$ et entre 14 et $15 \mathrm{~h}$.

Un biorythme circadien de la capacitance n'a été mis en évidence que chez $3 / 8$ volontaires (Tableau 2). Cependant, l'effet de l'heure était significatif $(p<0,0001)$ pour l'ensemble

Tableau 2

Évaluations de la présence de cycles chronobiologiques sur le cuir chevelu de huit sujets

\begin{tabular}{llllll}
\hline Sujet & \multicolumn{5}{l}{ Paramètre } \\
\cline { 2 - 6 } & a* & Capacitance & PIE & $\mathrm{pH}$ & $\begin{array}{l}\text { Débit de } \\
\text { sébum }\end{array}$ \\
\hline 1 & $\mathrm{NS}$ & $\mathrm{NS}$ & $* * *$ & $*$ & $* * *$ \\
2 & $* *$ & $* *$ & $*$ & $*$ & $* * *$ \\
3 & $*$ & $\mathrm{NS}$ & $* * *$ & $\mathrm{NS}$ & $* * *$ \\
4 & $* *$ & $\mathrm{NS}$ & $\mathrm{NS}$ & $* * *$ & $\mathrm{NS}$ \\
5 & $\mathrm{NS}$ & $*$ & $*$ & $\mathrm{NS}$ & $*$ \\
6 & $\mathrm{NS}$ & $* *$ & $* * *$ & $\mathrm{NS}$ & $* *$ \\
7 & $\mathrm{NS}$ & $\mathrm{NS}$ & $\mathrm{NS}$ & $* * *$ & $\mathrm{NS}$ \\
8 & $*$ & $\mathrm{NS}$ & $\mathrm{NS}$ & $\mathrm{NS}$ & $* *$ \\
\hline NS : non significatif $; * p \leq 0,05, * *$ & $* \leq 0,01, * * *$ & $p \leq 0,001$.
\end{tabular}

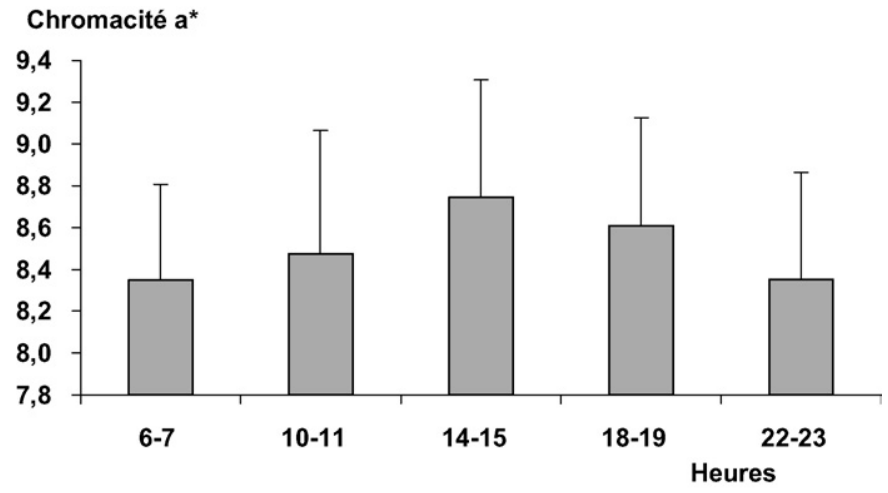

Fig. 1. Moyenne et écart-type de la valeur a* du cuir chevelu au cours de la période d'éveil.

du groupe. Aucune influence significative des jours n'a été observée. Pour la période observée du nycthémère, l'acrophase de veille $(73,35 \pm 2,30)$ se situait entre 14 et $15 \mathrm{~h}$, et le nadir $(70,30 \pm 2,30)$ entre 6 et 7 h (Fig. 2). Cette acrophase était $2,3 \%$ supérieure au mésor. La moyenne des $\mathrm{V}$ intraindividuels et interjournaliers variait de 1,88 à $2,99 \%$ selon les heures, le maximum étant atteint entre 18 et $19 \mathrm{~h}$.

Un rythme chronobiologique de la PIE a été observé chez $5 / 8$ sujets (Tableau 2). Pour l'ensemble du groupe, l'effet de l'heure s'est confirmé $(p<0,0001)$ alors qu'aucune influence des jours n'est apparue significative. L'acrophase de veille $\left(9,40 \pm 1,02 \mathrm{~g} / \mathrm{m}^{2}\right.$ par heure $)$ était retrouvée entre 14 et $15 \mathrm{~h}$ et le nadir $(8,47 \pm 0,70)$ entre 22 et $23 \mathrm{~h}$ (Fig. 3). L'acrophase était $5,4 \%$ supérieure au mésor. La moyenne des $\mathrm{V}$ intraindividuels et interjournaliers variait de 3,85 à 5,97\% selon les heures, le maximum étant atteint entre 14 et $15 \mathrm{~h}$.

Un rythme chronobiologique du pH a été décelé chez $4 / 8$ patients. Pour l'ensemble du groupe, l'effet du temps s'est avéré significatif $(p<0,001)$, tant dans les différences selon les jours que les heures. En traçant toutefois le plexogramme sur la période envisagée du nycthémère, il semblerait qu'un nadir se situe entre 22 et $23 \mathrm{~h}$ (Fig. 4).

Le débit sébacé instantané était rythmé chez $6 / 8$ sujets. Pour l'ensemble du groupe. L'effet heure était significatif $(p<0,001)$. Malgré des différences interjournalières $(p<0,001)$ dans l'intensité du flux sébacé chez certains individus, le rythme était synchrone d'un jour à l'autre. Pour la

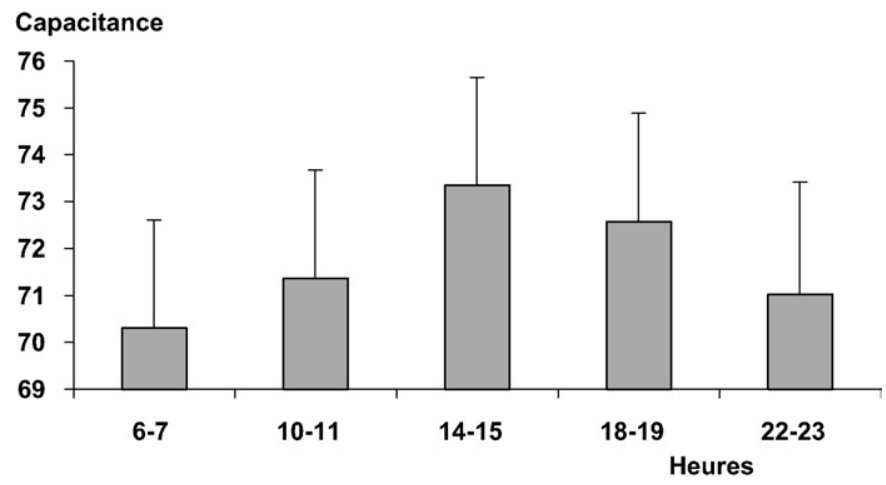

Fig. 2. Moyenne et écart-type de la valeur de la capacitance du cuir chevelu au cours de la période d'éveil. 


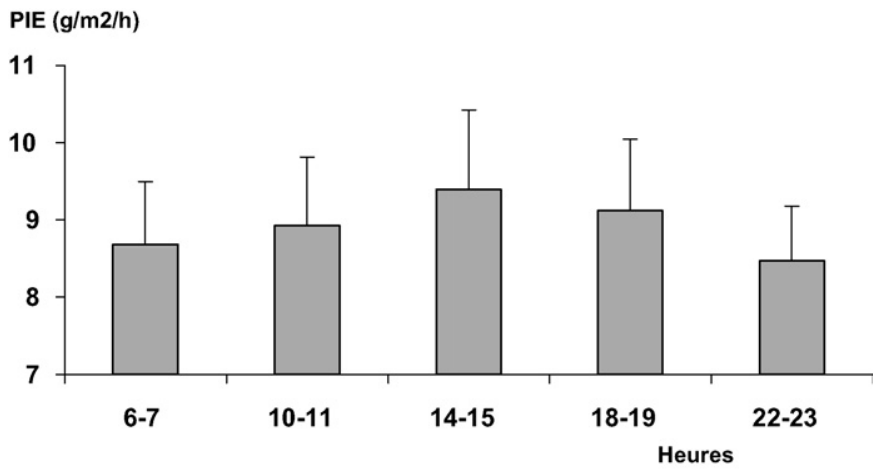

Fig. 3. Moyenne et écart-type de la valeur de la perte insensible d'eau (PIE) du cuir chevelu au cours de la période d'éveil.

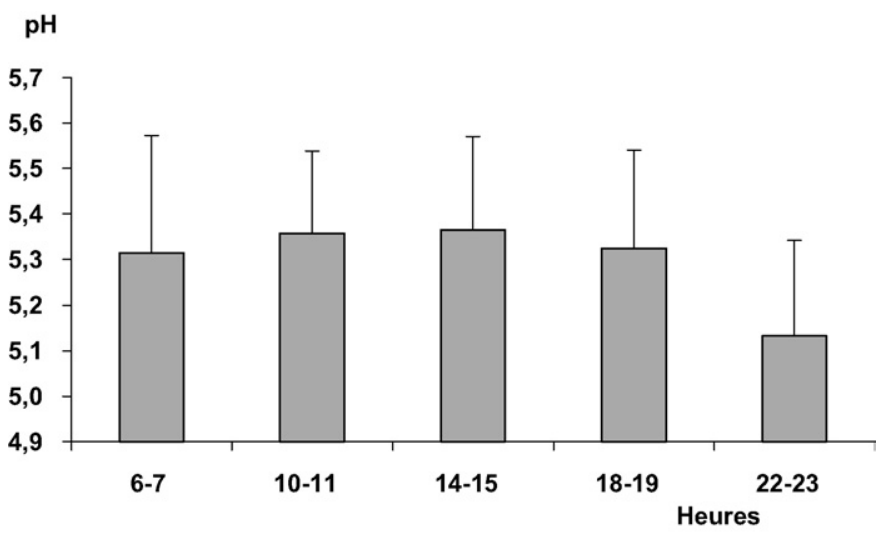

Fig. 4. Moyenne et écart-type de la valeur du pH du cuir chevelu au cours de la période d'éveil.

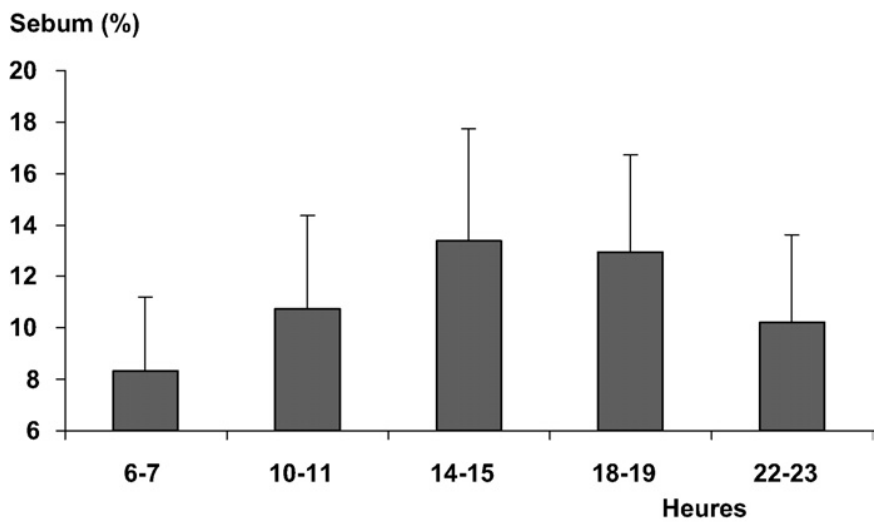

Fig. 5. Moyenne et écart-type du débit instantané de sébum (\%) au niveau du cuir chevelu au cours de la période d'éveil.

période étudiée du nycthémère, l'acrophase de veille $(13,38 \pm 4,36)$ se situait dans l'intervalle entre 14 et $15 \mathrm{~h}$ et le nadir $(8,31 \pm 2,88 \%)$ entre 6 et $7 \mathrm{~h}$ (Fig. 5). L'acrophase était $20,4 \%$ supérieure au mésor. La moyenne des $\mathrm{V}$ intraindividuels et interjournaliers variait de 15,61 à $21,77 \%$ selon les heures, le maximum étant atteint à l'acrophase du débit instantané de sébum.

Les variations percentuelles par rapport au mésor n'ont pas contribué à mieux percevoir les biorythmes.

\section{Discussion}

Des interactions existent entre les oscillateurs et les synchroniseurs. Chez l'homme, le synchroniseur externe le plus puissant est la lumière, et les synchroniseurs internes majeurs sont la mélatonine et la température corporelle. Un grand nombre des fonctions métaboliques sont gouvernées par des biorythmes. L'alternance d'expositions à la lumière et à l'obscurité, ainsi que le rythme nycthéméral endogène corollaire médié par la mélatonine, sont les principaux facteurs régulateurs [12]. De plus, il existe des structures internes permettant d'anticiper certaines variations périodiques de l'environnement. Dans ces conditions, les synchroniseurs sont confrontés aux mécanismes endogènes capables de se régler spontanément sur les phases du nycthémère. Les oscillateurs assurent la coordination entre les différents changements internes de l'organisme, tout en étant eux-mêmes synchronisés par l'environnement, de telle sorte que la période de l'horloge endogène est ajustée en permanence aux rythmes environnementaux.

Dans la présente étude, des biorythmes statistiquement significatifs ont été mis en évidence pour un ensemble de paramètres physiologiques du cuir chevelu. Cette constatation ne signifie cependant pas que ces fluctuations ont un impact important sur le plan physiologique. Il faut remarquer que tous les sujets, sans exception, ont démontré la présence d'un ou de plusieurs biorythmes dont le secteur temporel compris entre 14 et $15 \mathrm{~h}$ semble être un moment privilégié du cycle. Cela suggère la présence d'un oscillateur commun. Cependant, la majorité des sujets sont des non-répondeurs cycliques pour certains des paramètres évalués. Cette constatation pourrait suggérer une réactivité périphérique moindre des structures conditionnant ces paramètres physiologiques particuliers. Un effet perturbateur de synchroniseurs environnementaux est également possible.

L'excrétion de sébum est le paramètre qui a été le plus fréquemment retrouvé être soumis à un biorythme au niveau du visage $[17,19,20,25,26]$. Nous retrouvons un débit instantané de sébum qui est similaire au niveau du cuir chevelu. De plus, son amplitude est la plus élevée de toutes nos évaluations. Selon la physiologie complexe du follicule sébacé [31-33], ce phénomène ne peut être expliqué que par des modifications temporelles de la rhéologie du sébum à la sortie de l'infundibulum pilosébacé. L'intervention d'un synchroniseur de type température cutanée, elle-même sous l'influence de celle de l'environnement, est concevable. Cette dernière doit être évoquée puisqu'une différence significative a été retrouvée selon les jours successifs.

L'existence d'un rythme circadien de la PIE sur le visage et sur les avant-bras reste débattue [18,20,25,26,34]. Dans notre étude sur le cuir chevelu, il apparaît que le biorythme circadien de la PIE avait une ampleur importante, quoique inférieure à celle de l'excrétion instantanée du sébum. Ici aussi, la température cutanée pourrait jouer un rôle. Les biorythmes observés pour ces deux paramètres confortent la nécessité de fixer des horaires de mesure, standardisation déjà préconisée dans les recommandations du groupe EEMCO. 
Chacun des autres paramètres considérés, à savoir la capacitance, la valeur a* et le $\mathrm{pH}$, a montré un biorythme d'ampleur modeste dont la pertinence physiologique au niveau du cuir chevelu doit vraisemblablement être très limitée.

\section{Conclusion}

La chronobiologie et la chronopharmacologie sont des domaines en pleine évolution qui peuvent s'appliquer à une meilleure connaissance de la peau saine et des dermatoses. La peau a en effet ses horloges et ses rythmes qui s'égrainent au fil des mois, des jours et des heures. Les respecter devrait permettre une optimisation de certains traitements. Il existe des différences locorégionales sur le corps dans la nature et l'amplitude des biorythmes cutanés. Notre étude portant sur des individus synchronisés par un éveil diurne entre 6 et $23 \mathrm{~h}$ et un repos nocturne montre que le cuir chevelu est le siège de biorythmes circadiens. Pour ce territoire cutané particulier, les deux paramètres qui semblent les plus pertinents à envisager sur le plan clinique sont la rhéologie du sébum et la PIE. Les biorythmes observés pour ces deux paramètres confortent la nécessité de fixer des horaires de mesure, standardisation déjà préconisée dans les recommandations du groupe EEMCO.

\section{Remerciements}

Nous remercions le Pr A. Albert et Mme A.-F. Donneau du service de biostatistique de l'université de Liège pour leur aide précieuse dans l'évaluation statistique des résultats.

\section{Références}

[1] Henry F, et al. La peau et son horloge chronobiologique au quotidien. Rev Med Liege 2002;57:661-5.

[2] Flagothier C, et al. L'horloge chronobiologique de la peau. Dermatol Actual 2004;80:5-8.

[3] Ticher A, et al. Preservation of the functional advantage of human time structure. FASEB J 1995;9:269-72.

[4] Rusak B, Zucker I. Neural regulation of circadian rhythms. Physiol Rev 1979;59:449-56.

[5] Pittendrigh CS. Temporal organization: reflection of a Darwinian clockwatcher. Annu Rev Physiol 1993;55:16-54.

[6] Piérard-Franchimont $\mathrm{C}$, et al. Seasonal modulation of the sebum excretion. Dermatologica 1990;181:21-2.

[7] Piérard GE, Piérard-Franchimont C. L'effluvium télogène actinique : une facette de la chronobiologie humaine. Int J Cosmet Sci 1999;21:15-21.

[8] Tokumura F, et al. Seasonal variation in adhesive tape stripping of the skin. Skin Res Technol 1999;5:208-12.

[9] Black D, et al. Seasonal variability in the biophysical properties of stratum corneum from different anatomical sites. Skin Res Technol 2000;6: $70-6$.

[10] Burton JL, et al. Variations in sebum excretion during the menstrual cycle. Acta Derm Venereol 1973;53:81-4.

[11] Piérard-Franchimont $C$, et al. Rhythm of sebum excretion during the menstrual cycle. Dermatologica 1991;182:211-3.
[12] Reinberg A. La chronobiologie cutanée, importante à reconnaître et à respecter. Cosmetologie 2000;26:32-4.

[13] Fischer LB. The diurnal mitotic rhythm in the human epidermis. Br J Dermatol 1969;80:75-80.

[14] Zagula-Mally Z, et al. Time point differences in skin mitotic activity of actinic keratosis and skin cancers. Circadian reference, plasma cortisol. In: Reinberg A, Halberg F, editors. Chronopharmacology. Oxford: Pergamon Press; 1979. p. 399-402.

[15] Focan C, Piérard GE. Variations circadiennes de la prolifération cellulaire dans des tumeurs épidermiques. Bull Soc Fr Dermatol Syphil 1976;83:331-4

[16] Reinberg A, et al. Circadian changes in the size of facial skin corneocytes of healthy women. Ann Rev Chronopharmacol 1990;7:331-4.

[17] Burton JL, et al. Circadian rhythm in sebum excretion. Br J Dermatol 1979;82:497-502.

[18] Spruit D. The diurnal variation of water vapor loss from the skin in relation to temperature. Br J Dermatol 1971;84:66-70.

[19] Cotterill JA, et al. Variation in skin surface lipid composition and sebum excretion rate with time. Acta Derm Venereol 1973;53:271-4.

[20] Verschoore M, et al. Circadian variations in the number of actively secreting sebaceous follicles and androgen circadian rhythms. Chronobiol Int 1993;10:349-59.

[21] Touitou Y, et al. Circadian rhythms in a set of biochemical and biophysical skin variables (including transepidermal water loss) documented with non-invasive methods in healthy young women. In: Haus E, editor. Biological rhythms and medications. 1994 (XI-5).

[22] Yosipovitch G, et al. Time-dependent variations of the skin barrier function in humans: transepidermal water loss, stratum corneum hydration, skin surface pH, and skin temperature. J Invest Dermatol 1998;100:20-3.

[23] Chilcott RP, Farrar R. Biophysical measurements of human forearm skin in vivo: effects of site, gender, chirality and time. Skin Res Technol 2000;6:64-9.

[24] Burry JS, et al. Erroneous gender differences in axillary skin surface/ sweat pH. Int J Cosmet Sci 2001;23:99-107.

[25] Le Fur I, et al. Analysis of circadian and ultradian rhythms of skin surface properties of face and forearm of healthy women. J Invest Dermatol 2001;117:718-24.

[26] Latreille J, et al. Daily variations in skin surface properties using mixed model methodology. Skin Pharmacol Physiol 2004;17:133-40.

[27] Berardesca E, et al. EEMCO guidance for the assessment of stratum corneum hydration : electrical methods. Skin Res Technol 1997;3:126-32.

[28] Piérard GE. EEMCO guidance for the assessment of skin colour. J Eur Acad Dermatol Venereol 1998;10:1-11.

[29] Piérard GE, et al. EEMCO guidance for the in vivo assessment of skin greasiness. Skin Pharmacol Appl Skin Physiol 2000;13:372-89.

[30] Rogiers V, et al. EEMCO guidance for the assessment of the transepidermal water loss (TEWL) in cosmetic sciences. Skin Pharmacol Appl Skin Physiol 2001;14:117-28.

[31] Parra JL, et al. EEMCO guidance for the in vivo assessment of skin surface pH. Skin Pharmacol Appl Skin Physiol 2003;16:188-202.

[32] Piérard-Franchimont C, et al. Sebum rheology evaluated by two methods in vivo. Split-face study of the effect of a cosmetic formulation. Eur J Dermatol 1999;9:455-7.

[33] Saint-Léger D. Fonction sébacée normale et pathologique. Des recherches au milieu du gué ? Pathol Biol 2003;51:275-8.

[34] Reinberg A, et al. Oral contraceptives alter circadian rhythm parameters of cortisol, melatonin, blood pressure, heart rate, skin blood flow, transepidermal water loss and skin amino acids of healthy young women. Chronobiol Int 1996;13:199-211. 